\title{
High-throughput measurement of gap junctional intercellular communication
}

\author{
Jun Liu, ${ }^{1}$ Vinayakumar Siragam, ${ }^{2}$ Jun Chen, ${ }^{1}$ Michael D. Fridman, ${ }^{2}$ Robert M. Hamilton, ${ }^{2 *}$ and Yu Sun ${ }^{1 *}$ \\ ${ }^{1}$ Advanced Micro and Nanosystems Laboratory, University of Toronto, Toronto, Ontario, Canada; and ${ }^{2}$ Division of \\ Cardiology, the Hospital of Sick Children, Toronto, Ontario, Canada
}

Submitted 18 February 2014; accepted in final form 15 April 2014

Liu J, Siragam V, Chen J, Fridman MD, Hamilton RM, Sun Y. High-throughput measurement of gap junctional intercellular communication. Am J Physiol Heart Circ Physiol 306: H1708-H1713, 2014. First published April 28, 2014; doi:10.1152/ajpheart.00110.2014.— Gap junctional intercellular communication (GJIC) is a critical part of cellular activities and is necessary for electrical propagation among contacting cells. Disorders of gap junctions are a major cause for cardiac arrhythmias. Dye transfer through microinjection is a conventional technique for measuring GJIC. To overcome the limitations of manual microinjection and perform high-throughput GJIC measurement, here we present a new robotic microinjection system that is capable of injecting a large number of cells at a high speed. The highly automated system enables large-scale cell injection (thousands of cells vs. a few cells) without major operator training. GJIC of three cell lines of differing gap junction density, i.e., HeLa, HEK293, and HL-1, was evaluated. The effect of a GJIC inhibitor (18- $\alpha$-glycyrrhetinic acid) was also quantified in the three cell lines. System operation speed, success rate, and cell viability rate were quantitatively evaluated based on robotic microinjection of over 4,000 cells. Injection speed was 22.7 cells per min, with $95 \%$ success for cell injection and $>90 \%$ survival. Dye transfer cell counts and dye transfer distance correlated with the expected connexin expression of each cell type, and inhibition of dye transfer correlated with the concentration of GJIC inhibitor. Additionally, real-time monitoring of dye transfer enables the calculation of coefficients of molecular diffusion through gap junctions. This robotic microinjection dye transfer technique permits rapid assessment of gap junction function in confluent cell cultures.

robotic microinjection; automation; gap junctional intercellular communication; dye transfer

GAP JUNCTIONAL intercellular communication (GJIC) exists in many mammalian tissues. The gap junction is a specialized intercellular channel formed by the juxtaposition of two half channels called connexons $(14,16)$. Gap junctions are critical to several physiological roles, including impulse propagation in cardiac and neuronal tissue (4), regulation of embryonic development (19), and regulation of cellular proliferation (22). The abnormal GJIC is one major mechanism for cardiac arrhythmias $(12,33)$, which are a leading cause of cardiac morbidity and sudden death (5). The exchange of molecules through gap junctions includes the passive diffusion of small $(<1 \mathrm{kDa})$ and hydrophilic molecules, such as metabolites, nutrients, and second messengers (e.g., trisphosphate inositol and calcium) (2).

GJIC is regulated by the number of channels in the membrane, the functional state of gap junctions, and their permeability (15). The measurement of GJIC has been studied for decades by using a number of methods, such as dye transfer

\footnotetext{
* R. Hamilton and Y. Sun are co-principal investigators.

Address for reprint requests and other correspondence: Y. Sun, Univ. of Toronto, 5 King's College Rd., Toronto, ON, M5S 3G8, Canada (e-mail: sun@mie.utoronto.ca).
}

through microinjection (10), the scrape/scratch method (9), electroporation (29), fluorescence redistribution after photobleaching (36), and conductance measurement by dual-patch clamp $(24,26)$. Among these techniques, microinjection of membrane-impermeable, nontoxic tracers into single cells has been the most commonly used technique for identifying and mapping GJIC for a wide variety of cells (1). The microinjection method is considered superior to other techniques because of the following reasons: 1 ) microinjection permits the correlation of morphological and functional data from individual cells; 2) the technique enables kinetic studies aimed at evaluating the transfer rate from one cell to another; and 3) in microinjection, the level of cell communication is expressed as number of dye-coupled cells, permitting the direct comparison of GJIC in different cell types (18).

However, microinjection has stringent skill requirements, low cell viability rate, and poor reproducibility (7). Manual microinjection is typically used for injecting a few or tens of cells per experiment, limiting its usefulness when a large number of cells need to be tested for GJIC assessment. Here we report a robotic microinjection system and technique, for the first time, to enable the injection of hundreds of cells per experiment rapidly and accurately. The automated system is operated via computer mouse clicking for indicating target cells for microinjection. Training a user who has no skills in microinjection takes $\sim 15 \mathrm{~min}$, and after a few hours of operation, the user can readily become proficient at operating the system to perform microinjection with high success rates. In this report, system operation speed, microinjection success rate, and postinjection cell survival rate were quantitatively described, based on the injection of over 4,000 cells. With the high-throughput capability and high reproducibility of the system, GJIC of HeLa cells, HEK293 cells, and HL-1 cells was quantitatively measured, as these cell lines are known to have absent, moderate, or high expression of gap junctions, respectively. Finally, the effect of a GJIC inhibitor was tested in the three cell lines.

\section{METHODS}

Instruments. As shown in Fig. $1, A$ and $B$, the robotic injection system is built on a standard inverted microscope (Nikon Ti) with motorized magnification and motorized focusing control. An $X-Y$ motorized stage (ProScan, Prior), which has a travel range of $75 \mathrm{~mm}$ and a resolution of $0.01 \mu \mathrm{m}$ along both axes, is mounted on the microscope. A four degree-of-freedom micromanipulator controls the motion of the injection micropipette with a positioning resolution of $0.1 \mu \mathrm{m}$ along each axis. An injection micropipette pulled from a glass capillary is used to deliver fluorescent dyes into cells. The micropipette is connected to a digitally controlled pump (XenoWorks Digital Microinjector, Sutter Instrument). A camera is connected to the microscope to provide microscopy imaging and visual feedback. A host computer runs our custom-built control software to control all the aforementioned instruments. 


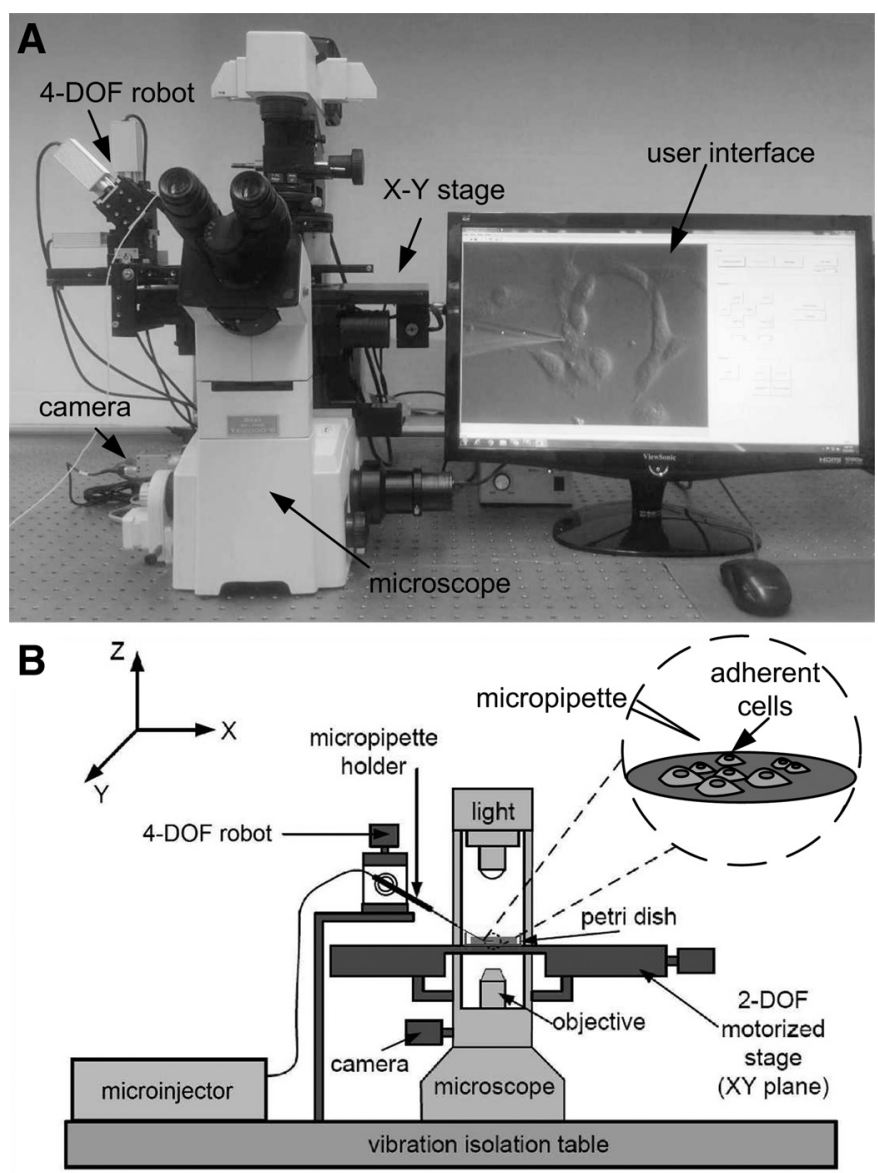

Fig. 1. A: automated robotic microinjection system. 4-DOF, four degrees of freedom. $B$ : schematic demonstration of the robotic microinjection system.

Autolocating micropipette tip. In manual microinjection, the operator needs to first locate the micropipette tip by operating the micromanipulator and microscope. Locating a micropipette tip that has a submicrometer diameter can be a challenge because the fragile tip is easy to break when colliding with other devices (e.g., petri dish). To help less-experienced microinjection operators avoid micropipette breakage, micropipette tip is located automatically in this robotic microinjection system. After the operator places the micropipette tip approximately within a $4 \mathrm{~mm}$-cube workspace, the robotic system automatically locates the micropipette tip under lower magnification (e.g., $\times 4$ ). Once the micropipette tip is detected under low magnification, the microscope is switched to higher magnifications, and the locating process is repeated.

The process of locating micropipette tip consists of two steps: detection of micropipette tip and autofocusing adjustment. In the detection step, the micropipette is moved in a zigzag pattern in the $X-Y$ plane. In the meanwhile, the system detects the micropipette tip's presence in the field of view (FOV) via computer vision. When the micropipette tip is present in the FOV, it is often partially or entirely out of focus. In the autofocusing step, therefore, the coarse and fine focus adjustments are performed to focus on the micropipette tip. Coarse adjustment moves the focal plane in a large step size until the focus measure (34) of the entire image reaches the maximum value. The fine focus adjustment divides the entire image into small regions $(10 \times 10$ pixel $)$ and finds the most in-focus region by calculating focus measure for each region. If the most in-focus region contains the micropipette body instead of the micropipette tip (i.e., partially in focus), the focal plane is moved downward until the micropipette tip is brought in focus. After the micropipette tip is focused, it is moved to the center of the FOV and close to the cell surface. Details on the detection and autofocusing of micropipette tips are described in Liu et al. (21).

Contact detection. After the micropipette tip is autolocated, the vertical distance between the micropipette tip and the cells must be determined. In the robotic system, two modes of contact detection are integrated to accurately determine the relative distance between micropipette tip and the cells along the $Z$ direction. One contact detection algorithm is to detect the micropipette tip's contact on the culture dish substrate when the cell confluency is low (i.e., there are empty areas on dish surface). The other algorithm is to detect the micropipette tip's contact on cell surface when cell confluency is high (e.g., fully confluent).

In contact detection on dish substrate (Fig. $2 A$ ), the system lowers the micropipette along the $Z$ direction when the system moves the $X-Y$ stage simultaneously. When the micropipette tip contacts the dish substrate, further vertical movement induces horizontal sliding on the dish substrate. Once the system detects the horizontal motion, the height of the micropipette tip is recorded in the system as the dish substrate's $Z$ position. In the subsequent microinjection step, the system inserts the micropipette tip into the cells at a vertical depth that is $2 \mu \mathrm{m}$ above the dish substrate. Details of contact detection on dish substrate are described in (38).

For detecting contact with the cell surface (Fig. $2 B$ ), the micropipette tip is first moved to the cell's position in the $X-Y$ plane. The micropipette is then moved downward to approach the cell surface along the $Z$-axis. When the end-effector tip contacts the cell surface, the cell is deformed and a subtle motion appears around the contact point, which is used to determine the cell's top surface position. In the subsequent microinjection step, the system inserts the micropipette tip into the cells at a vertical depth that is $2 \mu \mathrm{m}$ below the cell's top surface.

Robotic injection. In the software interface, the operator can select a single cell or multiple cells to inject by mouse clicking on the cells within the FOV. The system extracts cell templates centered at the mouse clicking positions. The templates are used for template matching to provide position feedback. After contact detection is performed, the system moves the micropipette tip close to the first target cell and inserts the micropipette into the cell along the tilting axis at the maximal speed (Fig. 2C). The pressure pump is then triggered to apply a positive pressure to deposit the preloaded material into the cell. Material deposition results in a "shock wave" motion inside the cell around the injection location (Fig. 2D). The volume of injected materials is precisely controlled by setting the injection pressure magnitude and width. Although target cells can be selected in a random order by the operator, the system injects selected cells along the shortest path. The system also records the locations of all injected cells, permitting time-lapsed imaging of cell response.

Injection volume control. Injection volume is determined by the injection pressure and pressure "on" time (i.e., pulse width). Injection volume was experimentally measured. The micropipette preloaded with distilled water was immersed into mineral oil. After 50 times of injection with a pressure "on" time of $0.4 \mathrm{~s}$, a water bubble was formed at the tip of the micropipette. The injection volume was then calculated based on the size of the water bubble. Figure $2 E$ shows the calibrated relationship between injection pressure and injection volume.

To keep a high postinjection survival rate, the volume of injected foreign materials must not exceed $5 \%$ of the cell's cytoplasmic volume (37), and therefore not exceed $30 \mathrm{fl}$ for most adherent cells. Accordingly, the injection pressure was set lower than $3,000 \mathrm{hPa}$ in our system. In addition to injection pressure, the pump also provides a constant positive pressure $(50 \mathrm{hPa})$ throughout the entire microinjection process. The application of this positive pressure ensures that there is a constant, gentle flow of solution from the micropipette, preventing undesired dilution of the solution in the micropipette by the medium inside the Petri dish. Moreover, the positive pressure also reduces the possibility of micropipette tip clogging by foreign objects in the surrounding medium. 
A
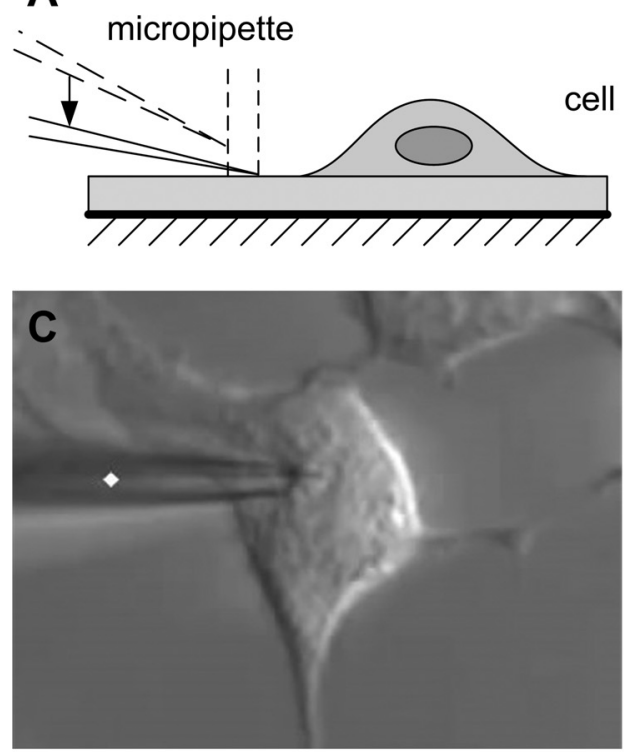

Fig. 2. A: schematic showing principle of contact detection on dish substrate. $B$ : schematic showing principle of contact detection on cell surface. $C$ : micropipette tip is inserted into an HL-1 cell. $D$ : materials are deposited into the same HL-1 cell. Scale bar $=20 \mu \mathrm{m} . E$ : injection volume control $F$ : dye transfer distance measurement.
B
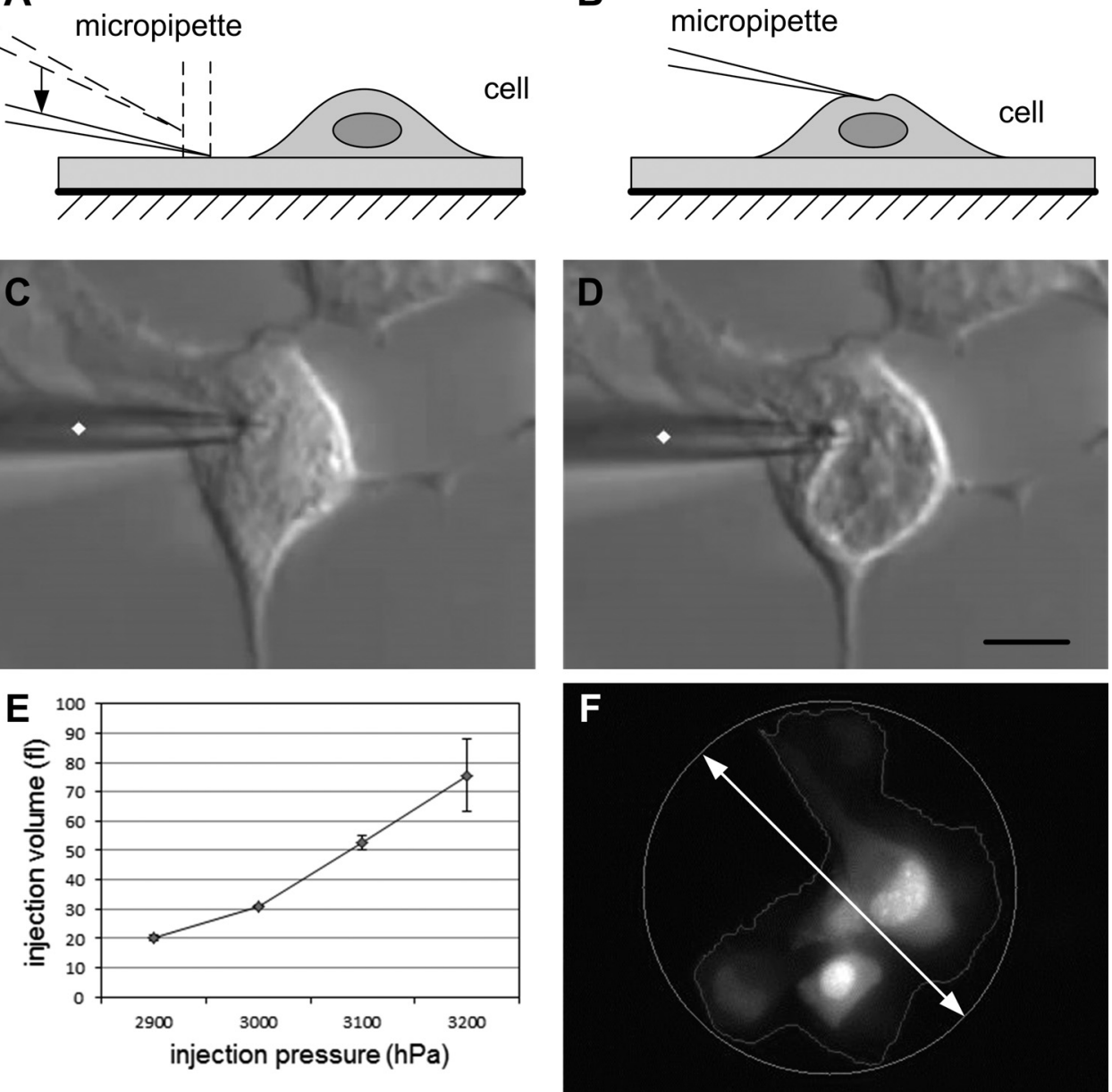

Dye transfer experiments. In the dye transfer experiments, the membrane-impermeable 8-hydroxypyrene-1,3,6-trisulfonic acid (HPTS) dye (Life Technologies, Burlington, CA), mixed with dextran-rhodamine dye (Life Technologies, Burlington, CA), was injected into a selected cell by using the robotic microinjection system. Both fluorescent dyes were at a concentration of $2 \mathrm{mM}$ and mixed at a ratio of $50 \%$. HPTS was chosen because of its small molecular size (molecular mass $=524.37 \mathrm{Da}$ ) and the membrane-impermeable property. HPTS is also highly water-soluble; therefore, it does not cause micropipette clogging.

After microinjection, HPTS molecules can be transferred from the injected cell to adjacent cells through gap junctions. In contrast, dextran-rhodamine molecules, because of their large size (molecular mass $=10,000 \mathrm{Da}$ ), cannot pass through gap junctions. Therefore, only the injected cells reveal red fluorescent color, whereas the injected cells as well as a number of adjacent cells reveal green fluorescent color (Fig. 3, $A-D$ ). GJIC was quantified by measuring the dye transferred cell number and dye transferred distance. The dye transferred cell number is the number of cells taking up the fluorescent molecule from the injected cell through gap junction. The dye transfer distance is the furthest distance that the fluorescent dye can be transferred.

GJIC was investigated for three cell lines (HeLa cells, Hek293 cells, and HL-1 cells). These cell lines have markedly different expression of connexin genes ( $\mathrm{Cx} 37, \mathrm{Cx} 40, \mathrm{Cx} 43$, and $\mathrm{Cx} 45)$ during their normal growth and differentiation. HL-1 atrial cardiomyocytes were maintained in Claycomb medium supplemented with $10 \%$ FCS, $100 \mu \mathrm{g} / \mathrm{ml}$ penicillin-streptomycin, $10 \mu \mathrm{M}$ epinephrine, $2 \mathrm{mM}$ and L-glutamine as previously described (6). HEK293 and Hela cells were cultured in DMEM supplemented with $10 \%$ fetal bovine serum at $37^{\circ} \mathrm{C}$ in $5 \% \mathrm{CO}_{2}-95 \%$ air humidified atmosphere. All three cell lines were seeded on $35-\mathrm{mm}$ petri dishes until reaching a high confluency $(>90 \%)$.

We also used 18- $\alpha$-glycyrrhetinic acid (18- $\alpha \mathrm{GA}$; Sigma-Aldrich) to alter GJIC in the three cell lines. This molecule is a pentacyclic triterpenoid derivative of the beta-amyrin type obtained from the hydrolysis of glycyrrhizic acid (11). It was used for blocking the roles of GJIC in fibroblast growth (23), myoblast fusion (25), and human trophoblast proliferation (27). In our experiments, the GJIC of three experimental groups with different doses $(25,50$, or $100 \mu \mathrm{M})$ of $18-\alpha$ GA treatment was measured. Two control groups were also included. Control group 1 (control 1 in Fig. 4A) had no 18- $\alpha$ GA. Since the $18-\alpha \mathrm{GA}$ stocking solution was dissolved in DMSO, a second control group (control 2) with only DMSO treatment was also examined.

Dye transfer measurement. After robotic microinjection, cells were washed with PBS solution (Life Technologies, Burlington, CA) and incubated for $5 \mathrm{~min}$. The cells were then placed back to the $X-Y$ stage on the system and automatically imaged under fluorescence imaging. Captured images were converted into grayscale and then binarized using an adaptive threshold algorithm. A minimum enclosing circle algorithm was then applied to bound the bright objects. The diameter of the bounding circle (see circle in Fig. $2 F$ ) was measured as the dye transfer distance.

The system also allows users to monitor fluorescent dye diffusion in real time. According to Safranyos et al. (31), diffusion kinetics is described by $C(r, t)=\left(s / 4 \pi D_{\mathrm{e}} h\right) E_{1}\left(r^{2} / 4 D_{\mathrm{e}} t\right)$, where $C(r, t)$ is the concentration of fluorescent dye at a distance $r$ from the injection 

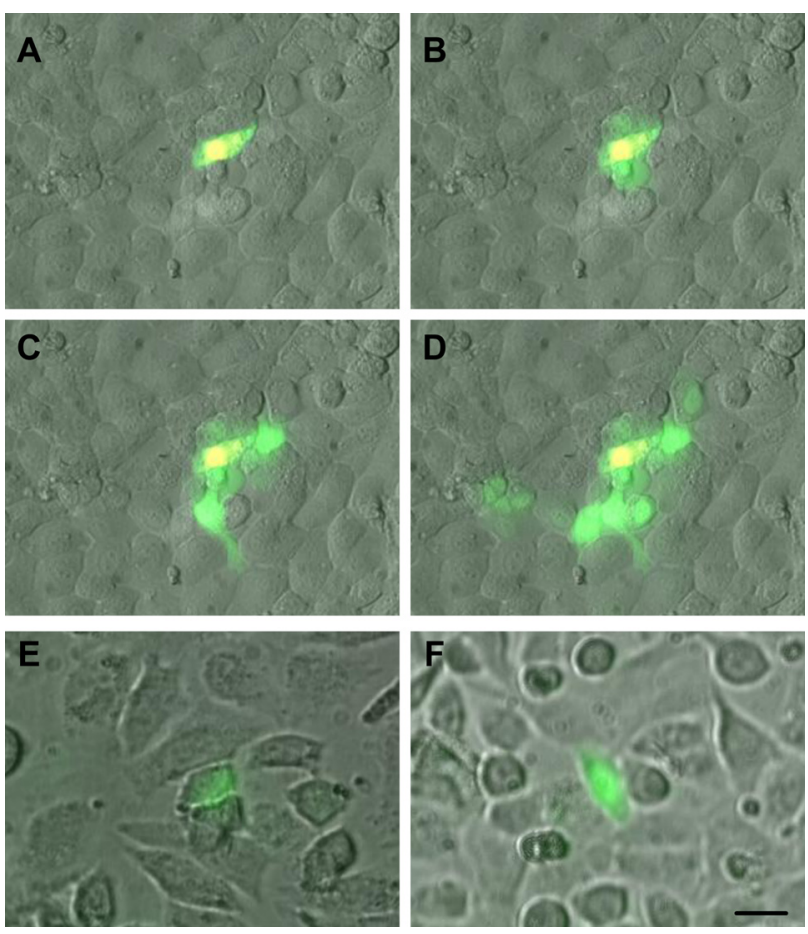

Fig. 3. $A-D$ : iime-lapsed images showing fluorescent dye is transferred from the injected HL-1 cell to adjacent cells at 50s, 100s, 150s, and 300s, respectively. $E$ and $F$ : HeLa cells do not show dye transfer through gap junctions. Scale bar $=20 \mu \mathrm{m}$.

location at time $t$ from the start of injection, $s$ is the dye injection rate, $h$ is the thickness of the monolayer cells, $E_{1}$ denotes the exponential integral, and $D_{\mathrm{e}}$ is the diffusion coefficient. Experimentally measured fluorescence intensity, which is proportional to its concentration, was fitted to this equation by using a nonlinear regression model. In this nonlinear regression model (32), the diffusion coefficient $D_{\mathrm{e}}$ is determined by dye transfer distance, time, and fluorescence intensity, independent of injection rate and cell thickness.

\section{RESULTS AND DISCUSSION}

Injection speed. Injection speed of the robotic system was evaluated by injecting the three cell lines. The number of injected cells and time consumed are summarized in Table 1. The injection time in Table 1 is the total experimental time for all steps including locating micropipette tip, contact detection, cell selection, and injection. The results from the injection of over 4,000 cells show that the injection speed of the robotic system is consistent across different cell lines. The average injection speed of 22.7 cells/min enables users to inject over a thousand cells within $1 \mathrm{~h}$ for assessing GJIC.

Injection success rate and cell survival rate. HPTS fluorescent dye was injected into the cytoplasm of HeLa cells for testing the success rate of robotic microinjection. After microinjection, the cells were examined under fluorescent microscope. Those cells revealing strong green fluorescent signals were counted and considered as successfully injected. Experimental results from injecting 1,245 cells show that the success rate was $95.2 \%$, which is significantly higher than previously reported injection success rates, $49.2 \%$ by Lim et al. (20), and $49 \%$ by Viigipuu and Kallio (35). This success rate improvement is mainly attributed to the system's capability of precisely
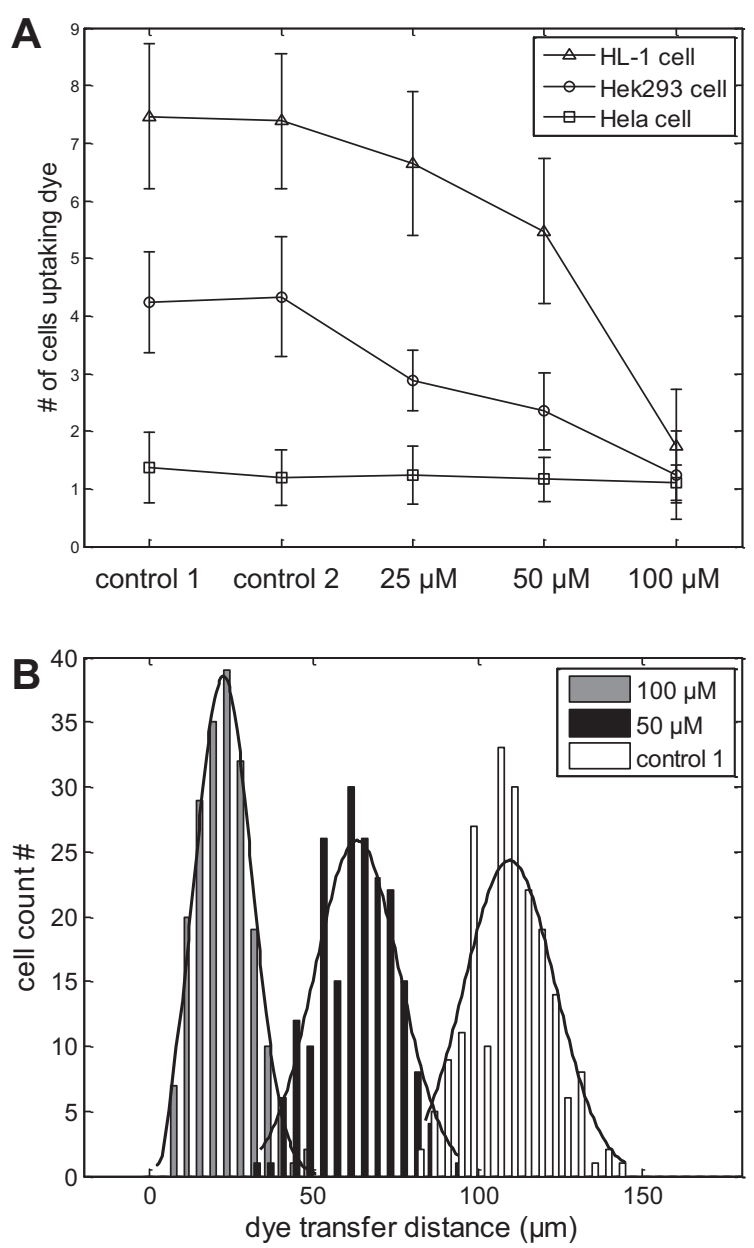

Fig. 4. Results of dye transfer experiments using the robotic microinjection system. A: number of dye transferred cells ( $n>200$ for each data point). $B$ : histogram of dye transfer distance for HL-1 cells.

detecting $Z$ positions of cells and micropipette tip and its capability of compensating for accumulative positioning errors.

Cell postinjection viability was evaluated for the three cell lines by using a cell viability assay kit (Viability/Cytotoxicity Kit, Life Technologies) after the injected cells were incubated for $2 \mathrm{~h}$. The measured cell survival rates for HeLa, HEK293, and HL-1 cells after microinjection were 97.2 $\pm 0.3,94.5 \pm$ 0.2 , and $93.7 \pm 0.4 \%$, respectively ( $n>100$ for each group; triplicate for each experiment). The results demonstrate that cells are able to maintain their viability at a high level $(>90 \%)$ for all the three adherent cell lines. Because of the embedded automated techniques in the robotic injection system, the injection success rate and cell survival rate are significantly increased, compared with other commercial joystick-based microinjection system $(20,35)$. Since the deposited materials in this study were fluorescence molecules (vs. DNA con-

Table 1. Robotic microinjection speed of three different cell lines

\begin{tabular}{lcccc}
\hline \hline & HeLa Cells & HEK293 Cells & HL-1 Cells & Overall \\
\hline Injected cell number & 2,281 & 1,068 & 1,116 & 4,465 \\
Injection time, min & 97 & 48 & 52 & 197 \\
Speed, cells/min & 23.5 & 22.3 & 21.5 & 22.7 \\
\hline
\end{tabular}


structs), the host cell's gene expression profiles should not have been changed. However, the effect of micropipette penetration and pressure application for material deposition on gene expression profiles still needs further study.

When beating HL-1 cells were injected, the beating cardiac muscle cells resumed their beating motion $\sim 1$ to $2 \mathrm{~s}$ after microinjection. This $\sim 1$ to $2 \mathrm{~s}$ interruption may have involved the reseal of cell membrane and the recovery from microinjection. For injecting the beating cardiac muscle cells, the contact detection on cell surface cannot be performed directly on the beating cell, because the beating motion will interfere with cell deformation-caused motion. In our experiments, we found that not all HL-1 cells beat, similar to the findings of others (13, 40). Therefore, the contact detection on cell surface was performed on a neighboring nonbeating cell that has a similar height with the target beating cells. In the experiments, both beating and nonbeating HL-1 cells are injected for GJIC measurement because they both express cardiac gap junction proteins (28).

Characterization of GJIC. The results from dye transfer experiments for testing the GJIC of three cell lines show that HL-1 cells (a cardiac muscle cell line) have much higher GJIC than the other two cell lines, as shown in Fig. 4A. GJIC plays a particularly important role in impulse propagation in cardiac tissue (30). When the impulse signal is passed efficiently through gap junctions, the cardiac tissue contracts and relaxes at the same tandem. In contrast, Hela cell, a cancer cell line derived from cervical cancer cells, is known to be intrinsically deficient in major gap junctions. This may be a factor in tumor formation, potentially inhibiting the intercellular GJIC within the cells (17). Human embryonic kidney (HEK) cells are known to express an intermediate amount of gap junctions.

The experimental data also shows that there is no significant difference between the two control groups. The number of adjacent cells taking up fluorescent dye from the injected cells significantly decreased with a higher dose of GJIC inhibitor. The results show that $100 \mu \mathrm{M}$ of $18-\alpha \mathrm{GA}$ effectively blocked almost all the gap junctions for both cell lines which express them. This suggests that the glycyrrhetinic acid derivatives potentially disturb GJIC by blocking gap junctions. The dye transfer distance was also measured for the HL-1 cells. With the robotic injection system, a number of cells (over 200 cells for each group) were injected for investigating the GJIC of HL-1 cells. The histogram in Fig. $4 B$ clearly shows that a higher dose of $18-\alpha \mathrm{GA}$ inhibitor resulted in significantly shorter dye transfer distances. To quantitatively measure the GJIC function, fluorescent dye diffusion coefficient was calculated. The diffusion coefficients of control group and $50-\mu \mathrm{M}$ treatment group were $3.62 \times 10^{-7}$ and $1.28 \times 10^{-7} \mathrm{~cm}^{2} / \mathrm{s}$, respectively. These diffusion coefficients are within the same order of magnitude as previously reported values for fluorescent dyes with similar molecular mass $(3,31,39)$. The diffusion coefficient differences also indicate that molecule diffusion through gap junctions was suppressed by $18-\alpha \mathrm{GA}$ treatment. The GJIC alteration may influence both chemical and electrical coupling (8). In addition to the measurement of chemical coupling by dye transfer, electrical coupling can be quantitatively measured by whole cell dual-voltage clamp.

Manual microinjection of adherent cells and existing robotic system prototypes are limited to the injection of a few to tens of cells per experiment at best. Our new robotic system described in this article is the first system capable of performing microinjection on hundreds and thousands of cells per experiment. The system is embedded with strong automation capabilities in every step of operation, enabling an operator to perform the entire microinjection process via computer mouse clicking in front of a computer monitor.

Conclusion. This article reported a robotic microinjection system with a high degree of automation. It enables users with no microinjection training to perform large-scale cell microinjection with high success rates. System operation speed, success rate, and cell viability rate were quantitatively evaluated. We demonstrate that the robotic microinjection system is capable of performing dye transfer experiments for characterizing the GJIC of different types of cells, using large sample sizes with a high reproducibility. The high-throughput system can also be useful for testing the efficacy of drugs that alter the GJIC of cells. The robotic injection system can also be possibly adapted for other applications, such as electrophysiological patch clamping.

\section{GRANTS}

Y. Sun acknowledges financial support from the Natural Sciences and Engineering Research Council of Canada (NSERC) through a discovery grant, from the University of Toronto through a connaught innovation award, and from the Canada Research Chairs Program. R. Hamilton acknowledges financial support from Canadian Institutes of Health Research Grant 102166 and generous donations from the Caitlin Morris Memorial Fund and Alex Corrance Memorial Fund to the Hospital for Sick Children Foundation.

\section{DISCLOSURES}

No conflicts of interest, financial or otherwise, are declared by the author(s).

\section{AUTHOR CONTRIBUTIONS}

J.L., M.D.F., R.M.H., and Y.S. conception and design of research; J.L., V.S., and J.C. performed experiments; J.L. and V.S. analyzed data; J.L., V.S., M.D.F., R.M.H., and Y.S. interpreted results of experiments; J.L. prepared figures; J.L. and V.S. drafted manuscript; J.L., R.M.H., and Y.S. edited and revised manuscript; J.L., V.S., J.C., R.M.H., and Y.S. approved final version of manuscript.

\section{REFERENCES}

1. Abbaci M, Barberi-Heyob M, Blondel W, Guillemin F, Didelon J. Advantages and limitations of commonly used methods to assay the molecular permeability of gap junctional intercellular communication. Biotechniques 45: 33-52, 56-62, 2008

2. Alexander DB, Goldberg GS. Transfer of biologically important molecules between cells through gap junction channels. Curr Med Chem 10: 2045-2058, 2003.

3. Bathany C, Beahm D, Felske JD, Sachs F, Hua SZ. High throughput assay of diffusion through $\mathrm{Cx} 43$ gap junction channels with a microfluidic chip. Anal Chem 83: 933-939, 2011.

4. Brink PR, Cronin K, Ramanan SV. Gap junctions in excitable cells. $J$ Bioenerg Biomembr 28: 351-358, 1996.

5. Chugh SS, Reinier K, Teodorescu C, Evanado A, Kehr E, Al Samara M, Mariani R, Gunson K, Jui J. Epidemiology of sudden cardiac death: clinical and research implications. Prog Cardiovasc Dis 51: 213-228, 2008.

6. Claycomb WC, Lanson NA Jr, Stallworth BS, Egeland DB, Delcarpio JB, Bahinski A, Izzo NJ Jr. HL-1 cells: a cardiac muscle cell line that contracts and retains phenotypic characteristics of the adult cardiomyocyte. Proc Natl Acad Sci USA 95: 2979-2984, 1998.

7. Czyz J, Irmer U, Schulz G, Mindermann A, Hülser DF. Gap-junctional coupling measured by flow cytometry. Exp Cell Res 255: 40-46, 2000.

8. Ek-Vitorin JF, Burt JM. Structural basis for the selective permeability of channels made of communicating junction proteins. Biochim Biophys Acta 1828: 51-68, 2013.

9. El-Fouly MH, Trosko JE, Chang CC. Scrape-loading and dye transfer. Exp Cell Res 168: 422-430, 1987. 
10. Fitzgerald DJ, Murray AW. Inhibition of intercellular communication by tumor-promoting phorbol esters. Cancer Res 40: 2935-2937, 1980.

11. Goldberg GS, Moreno AP, Bechberger JF, Hearn SS, Shivers RR, MacPhee DJ, Zhang YC, Naus CC. Evidence that disruption of connexon particle arrangements in gap junction plaques is associated with inhibition of gap junctional communication by a glycyrrhetinic acid derivative. Exp Cell Res 222: 48-53, 1996.

12. Gutstein DE, Danik SB, Lewitton S, France D, Liu F, Chen FL, Zhang J, Ghodsi N, Morley GE, Fishman GI. Focal gap junction uncoupling and spontaneous ventricular ectopy. Am J Physiol Heart Circ Physiol 289: H1091-H1098, 2005.

13. Guzun R, Karu-Varikmaa M, Gonzalez-Granillo M, Kuznetsov AV, Michel L, Cottet-Rousselle C, Saaremäe M, Kaambre T, Metsis M, Grimm M, Auffray C, Saks V. Mitochondria-cytoskeleton interaction: distribution of $\beta$-tubulins in cardiomyocytes and HL-1 cells. Biochim Biophys Acta 1807: 458-469, 2011.

14. Hertzberg EL, Lawrence TS, Gilula NB. Gap junctional communication. Annu Rev Physiol 43: 479-491, 1981.

15. Hervé JC, Bourmeyster N, Sarrouilhe D. Diversity in protein-protein interactions of connexins: emerging roles. Biochim Biophys Acta 1662: 22-41, 2004

16. Heyman NS, Kurjiaka DT, Ek Vitorin JF, Burt JM. Regulation of gap junctional charge selectivity in cells coexpressing connexin 40 and connexin 43. Am J Physiol Heart Circ Physiol 297: H450-H459, 2009.

17. Holder JW, Elmore E, Barrett JC. Gap junction function and cancer. Cancer Res 53: 3475-3485, 1993.

18. Juul MH, Rivedal E, Stokke T, Sanner T. Quantitative determination of gap junction intercellular communication using flow cytometric measurement of fluorescent dye transfer. Cell Adhes Commun 7: 501-512, 2000.

19. Kalimi GH, Lo CW. Gap junctional communication in the extraembryonic tissues of the gastrulating mouse embryo. J Cell Biol 109: 30153026, 1989.

20. Lim SN, Zeenathul NA, Mohd Azmi ML, Abas Mazni O, Fauziah O. Effect of protein concentration and injection pressure in microinjection delivery of maltose binding protein into breast cancer cells. Pertanika $J$ Sci Technol 19: 273-283, 2011.

21. Liu J, Gong Z, Tang K, Lu Z, Ru C, Luo J, Xie S, Sun Y. Locating end-effector tips in robotic micromanipulation. IEEE Trans Robot 30: 125-130, 2014.

22. Loewenstein WR. Junctional intercellular communication: the cell-to-cell membrane channel. Physiol Rev 61: 829-913, 1981

23. Martin W, Zempel G, Hülser D, Willecke K. Growth inhibition of oncogene-transformed rat fibroblasts by cocultured normal cells: relevance of metabolic cooperation mediated by gap junctions. Cancer Res 51: $5348-5351,1991$.

24. McCain ML, Desplantez T, Geisse NA, Rothen-Rutishauser B, Oberer H, Parker KK, Kleber AG. Cell-to-cell coupling in engineered pairs of rat ventricular cardiomyocytes: relation between $\mathrm{Cx} 43$ immunofluorescence and intercellular electrical conductance. Am J Physiol Heart Circ Physiol 302: H443-H450, 2012.
25. Mège RM, Goudou D, Giaume $\mathbf{C}$, Nicolet $\mathbf{M}$, Rieger $\mathbf{F}$. Is intercellular communication via gap junctions required for myoblast fusion? Cell Adhes Commun 2: 329-343, 1994.

26. Neyton J, Trautmann A. Single-channel currents of an intercellular junction. Nature 317: 331-335, 1985.

27. Nishimura T, Dunk C, Lu Y, Feng X, Gellhaus a Winterhager E, Rossant J, Lye SJ. Gap junctions are required for trophoblast proliferation in early human placental development. Placenta 25: 595-607, 2004.

28. Pelloux S, Robillard J, Ferrera R, Bilbaut A, Ojeda C, Saks V, Ovize M, Tourneur Y. Non-beating HL-1 cells for confocal microscopy: application to mitochondrial functions during cardiac preconditioning. Prog Biophys Mol Biol 90: 270-298, 2006.

29. Raptis LH, Brownell HL, Firth KL, Mackenzie LW. A novel technique for the study of intercellular, junctional communication: electroporation of adherent cells on a partly conductive slide. DNA Cell Biol 13: 963-975, 1994.

30. Rohr S. Role of gap junctions in the propagation of the cardiac action potential. Cardiovasc Res 62: 309-322, 2004.

31. Safranyos RG, Caveney S, Miller JG, Petersen NO. Relative roles of gap junction channels and cytoplasm in cell-to-cell diffusion of fluorescent tracers. Proc Natl Acad Sci USA 84: 2272-2276, 1987.

32. Safranyos RG, Caveney S. Rates of diffusion of fluorescent molecules via cell-to-cell membrane channels in a developing tissue. J Cell Biol 100: 736-747, 1985.

33. Strom M, Wan X, Poelzing S, Ficker E, Rosenbaum DS. Gap junction heterogeneity as mechanism for electrophysiologically distinct properties across the ventricular wall. Am J Physiol Heart Circ Physiol 298: H787H794, 2010.

34. Sun Y, Duthaler S, Nelson BJ. Autofocusing in computer microscopy: selecting the optimal focus algorithm. Microsc Res Tech 65: 139-149, 2004.

35. Viigipuu K, Kallio P. Microinjection of living adherent cells by using a semi-automatic microinjection system. Altern Lab Anim 32: 417-423, 2004.

36. Wade MH, Trosko JE, Schindler M. A fluorescence photobleaching assay of gap junction-mediated communication between human cells. Science 232: 525-528, 1986.

37. Wang W, Sun Y, Zhang M, Anderson R, Langille L, Chan W. A system for high-speed microinjection of adherent cells. Rev Sci Instrum 79: 104302, 2008

38. Wang WH, Liu XY, Sun Y. Contact detection in microrobotic manipulation. Int J Robot Res 26: 821-828, 2007.

39. Weber PA, Chang HC, Spaeth KE, Nitsche JM, Nicholson BJ. The permeability of gap junction channels to probes of different size is dependent on connexin composition and permeant-pore affinities. Biophys $J$ 87: 958-973, 2004.

40. Yang Z, Murray KT. Ionic mechanisms of pacemaker activity in spontaneously contracting atrial HL-1 cells. J Cardiovasc Pharmacol 57: $28-36,2011$. 